\title{
LOW-COMPLEX ADAPTIVE POST FILTER FOR ENHANCEMENT OF CODED VIDEO
}

\author{
Andreas Rossholm, Benny Lövström \\ Department of Signal Processing \\ School of Engineering \\ Blekinge Institute of Technology \\ Ronneby, Sweden
}

\author{
Kenneth Andersson \\ Multimedia Technologies \\ Ericsson Research \\ Ericsson AB \\ Kista, Sweden
}

\begin{abstract}
In this paper an adaptive filter that removes de-blocking and de-ringing artifacts and also enhances the sharpness of decoded video, which may be caused by zeroing highfrequency DCT coefficients, is presented. The solution is designed with consideration of Mobile Equipment with limited computational power and memory. Also, the solution is computationally scalable to be able to handle limited computational resources in different user cases. In the paper it is shown that the adaptive filter always keeps or increases the image quality, compared to the original decoded sequences, and that the amount of sharpening decreases with an decrease of bit-rate to limit amplification of coding artifacts or noise.
\end{abstract}

\section{INTRODUCTION}

In the Mobile Equipment (ME) today the use of video becomes more and more common. To make it possible to view a video clip or streaming video, or to make a video telephony call, it is important to compress the data as much as possible. Most video codecs, video encoder and decoder, used today are designed as a block-based motion-compensated hybrid transform coder, as MPEG-4, H.263, where the transformation is done by a Discrete Cosine Transforms (DCT) on blocks of 8x8 pixels. The DCT coefficients are quantized with a quantization parameter (QP). Two of the main artifacts from the quantization of the DCT are blocking and ringing [2]. To reduce blocking artifacts, two-dimensional (2D) low-pass filtering of pixels on block boundaries of the decoded image(s) was suggested in [3]. The 2D space-invariant static filtering described in that paper reduces blocking artifacts but can also introduce blurring artifacts when true edges in the image are low-pass filtered. To avoid blurring of true edges in the image and also to be computationally efficient, the amount of low-pass filtering may be controlled by tablelookup as described in [4]. Large differences between initial pixel values and filtered pixel values are seen as natural image structure, and thus filtering is weak so that the image is not blurred. Small pixel differences are seen as coding artifacts, and thus stronger filtering is allowed to remove the artifacts. Based on data from other equipment, the amount of filtering can be controlled by using additional filter tables. The algorithm modifies the output of a low-pass-filtered signal with the output of a table-lookup using the difference between a delayed input signal and the filtered signal as an index into the table, and different degrees of filtering are achieved only by providing additional tables. A combined de-blocking and de-ringing filter was proposed in [5]. The proposed filter used filter strengths on block boundaries that were different from filter strengths inside blocks, allowing for stronger filtering at block boundaries than inside blocks. This was achieved by using a metric that used different constants when computing the output values of block boundary pixels versus the output values of pixels inside the block boundary. The metric also included the QP value. These and most other current algorithms handle de-blocking and de-ringing artifacts sequentially. Such double filtering can have a negative impact on computational complexity and memory consumption, which are parameters of particular importance in many devices, such as mobile communication devices. Moreover, removal of blocking and ringing artifacts can add visually annoying blurring artifacts as described above. It is thus important to be careful with strong image features that likely are natural image features and not coding artifacts. In [6] an adaptive non-linear filter is proposed. The proposed filter handles both the coding artifacts and performs sharpening on true details. However, this filter uses a rational function for the control of the filter function based on measures of variance. This gives good results but is a too complex solution for implementation in a ME.

In this paper, we propose a filter that performs enhancement on the coded video stream including both de-blocking, de-ringing and sharpening based on the output from a reference filter, which requires much less computational power than the state of art approach. This filter is a further development of our adaptive de-blocking and de-ringing filter published in [1].

\section{THE ADAPTIVE FILTER}

The proposed filter is developed with two main considerations; limiting the computational complexity, and limiting the amount of working memory. The idea is to filter rows of pixels of an image in a vertical direction, store the results in row vectors, and then filter the row vectors in the horizontal direction, and display the results. In 
the following part the adaptive filter is described in one of the above directions. Coefficients of a reference filter are modified based on the output from the reference filter passed through a table-lookup process that accesses a table of modifying weight coefficients. The output of the modified filter is added to a delayed version of the input to provide the adaptive filter output. A block diagram of the adaptive filter is shown in Fig. 1.

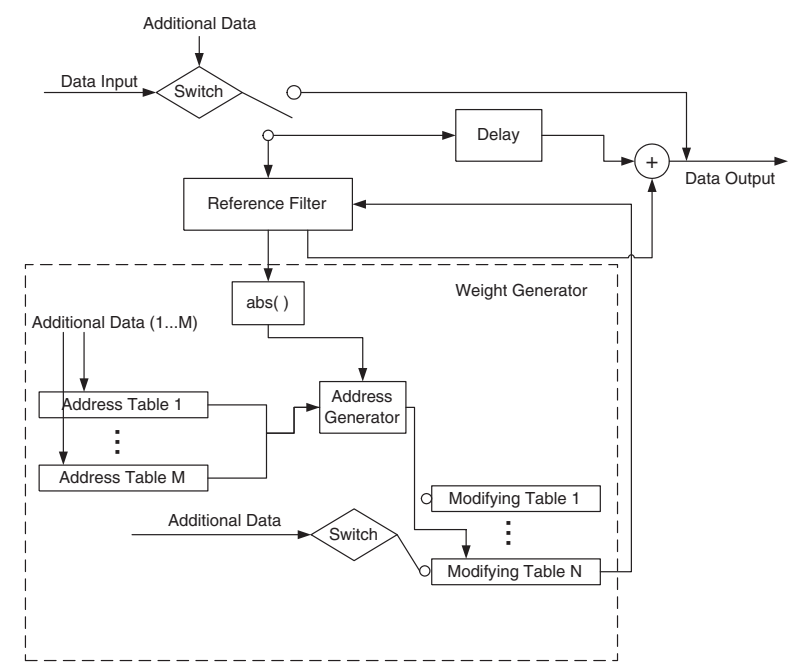

Fig. 1. A block diagram of the adaptive filter.

\subsection{Overview of the filter}

In Fig. 1 an input data stream of pixel data is provided to a switch that directs the input pixels to either the output of the filter or to a delay element and a reference filter. The operation of the switch is responsive to additional data, in particular, whether the input pixels belong to an error-concealed block or if the amount of filtering is limited based on location in the frame, as described in more detail below. The reference filter has coefficients that determine the filtering function, and these coefficients are selectively modified. The output of the reference filter is provided to an adder that combines the output with the delayed input produced by the delay element, thereby generating the output of the adaptive filter.

The modification of the output of the reference filter is performed by a weight generator that produces weights that selectively modify the coefficients of the filter based on the filter output to the weight generator. A signal corresponding to the absolute value of the reference filter output is produced, and this signal is provided to an address generator. The absolute value together with additional data provided by $M$ suitable address tables, generates addresses into $N$ tables of modifying weight coefficients, as described in more detail below. As a set of modifying weight coefficients is retrieved from the selected table, it is provided by the weight generator to the filter, and the transfer function of the reference filter is modified accordingly. Through this modification, the filter adapts to the input stream of pixels.

\subsection{Reference Filter}

In the adaptive filter a 5 -tap reference filter is used, $\left[\begin{array}{lll}1 & 1-\end{array}\right.$ $\left.\begin{array}{lll}4 & 1 & 1\end{array}\right]$. The number of filter taps chosen is the result of a trade-off between the amount of low-pass filtering that can be performed, locality in filtering, and computational complexity. The filter coefficients are chosen to detect variations in pixel value in the filter neighborhood with as low complexity as possible. The same filter is used for filtering luminance, denoted $Y$, and chrominance blocks, denoted $U$ and $V$, although luminance blocks are more important to filter than chrominance blocks. The modification of the reference filter is performed with a set of modifying weights which results in de-blocking/deringing or sharpening. If the modifying weights are such that all filter coefficients are modified in the same way, the output of the modified reference filter is simply a scaling of the output of the unmodified reference filter. Otherwise, the output of the modified reference filter is calculated using the input pixels and the modified reference filter transfer function.

\subsubsection{De-blocking and De-ringing}

For de-blocking and de-ringing the resulting adaptive filter response is illustrated in Fig. 2. It can be seen that

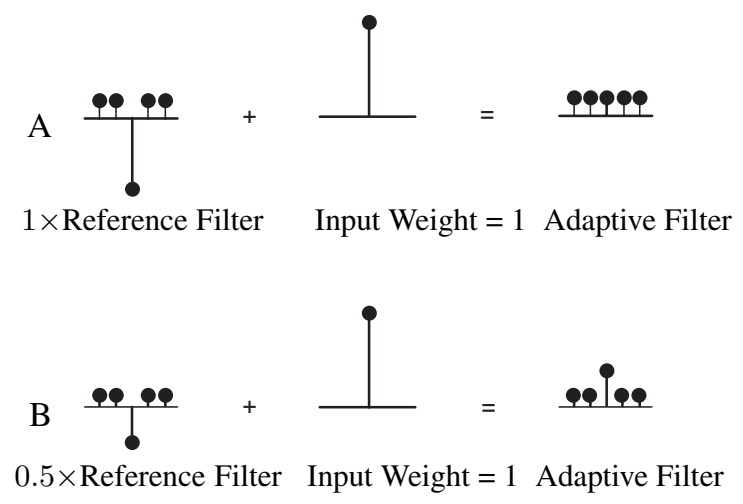

Fig. 2. Depiction of reference filter modification and adaptive filter response.

the same modification is made to each coefficient. In the figure, the sign and magnitude of a filter coefficient or a weight are indicated by the length of the respective vertical line segment and its position above or below the horizontal reference line. The " + " sign indicates the operation of the adder. In Fig. 2B the modifying weight is shown as 0.5 and the other coefficients are fixed. Comparing Fig. $2 \mathrm{~A}$ and Fig. 2B, it will be seen that a "weaker" adaptive filter is achieved when the reference filter coefficients are scaled by a factor of 0.5 , i.e., neighboring pixels have less influence on the modified-filter output for a pixel.

\subsubsection{Sharpening}

For sharpening the same concept as described above can be used by changing the sign of the reference filter, which generates a high-pass filter compared to the above-described low-pass filter. This is illustrated in FIG. 3. Comparing 
A

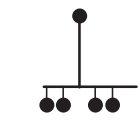

$-1 \times$ Reference Filter Input Weight $=1$ Adaptive Filter

B

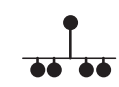

$-0.5 \times$ Reference Filter Input Weight $=1$ Adaptive Filter

Fig. 3. Depiction of reference filter modification and adaptive filter response.

this figure with FIG. 2 the difference between an adaptive sharpening, or high-pass, filter and an adaptive lowpass filter will be recognized. As in FIG. 2 the FIG. 3 depict how modification of the coefficients of the reference filter with a set of modifying weights modifies the adaptive filter response. In the case illustrated by the figure, the same modification is made to each coefficient. In FIG. 3A, the modifying weight is shown as -1 and the other coefficients are fixed. In FIG. 3B, the modifying weight is shown as -0.5 and the other coefficients are fixed. Comparing FIG. 3A and FIG. 3B, it will be seen that a "weaker" adaptive filter is achieved when the reference filter coefficients are scaled by a smaller negative factor, i.e., neighboring pixels have less influence on the modified-filter output for a pixel.

\subsection{Weight Generator}

The weight generator handles the adaptive part of the filter. It is divided into three main parts: The first part, address table, uses QP for the block as additional data and the address table length correspond to the range of the QP data. The output from the address table are positive for low QP values and negative for high QP values, resulting in potentially weaker and stronger filtering, respectively, depending on the magnitude of the reference filter output. Several address tables can be used if different sessions needs different strength of filtering.

The second part, address generator, produces a signal corresponding to the absolute value of the filter output, together with the output from the address tables, to generate addresses into one of the modifying tables with weight coefficients. This input also determines wether the filter is low-pass or high-pass, de-blocking/de-ringing or sharpening.

The third part, modifying tables, provides sets of weight coefficients to modify the transfer function of the reference filter, resulting in a modified, or adapted, transfer function for the adaptive filter as described in subsection 2.2. The length (i.e., the address range) of a modifying tables corresponds to the range of the reference filter output.

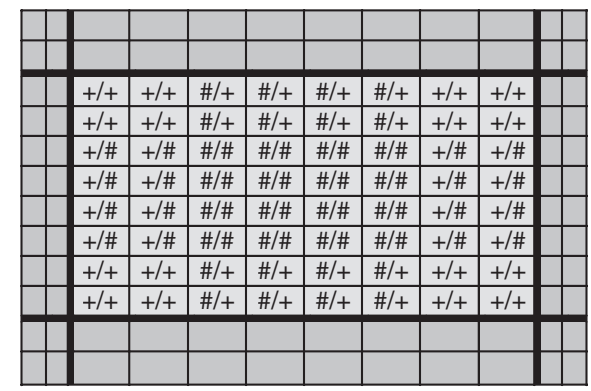

Fig. 4. Depiction of a block of pixels.

The additional data that is input to the switch, selecting modification table, is based on the position of a pixel in its block. A block of pixels is illustrated in FIG. 4 where the outer boundary pixels are indicated by + and the inner pixels are indicated by \#. Also, the $x / y$ in FIG. 4 describes filtering in horizontal/vertical direction.

In the de-blocking and de-ringing case stronger filtering is performed on boarder pixels than the table selected for inner block pixels. Furthermore, the weights of the selected table for de-blocking and de-ringing decreases more quickly with increasing index than the weights in the boundary pixels table. This results in reduction of blocking and ringing artifacts without blurring the image too much. For sharpening more weights are given to the inner pixels and thereby sharpening central parts of the block with normally less prominent coding artifacts. The block boundary pixels are not sharpened at all or only weakly sharpened to avoid amplifying block artifacts.

The resulting filter characteristics can hereby vary with the absolute value of the reference filter output by using QP as additional data in tables and position of a pixel in its block gradually changing from strong low-pass filtering (large positive weight) to strong high-pass filtering (large negative weight) when the reference filter output magnitude is relatively larger.

\subsection{Further considerations}

The first switch in Fig.1 makes it possible to limit the amount of filtering for different combinations of applications for a given device. The priority of filtering is given from low to high priority as, all luminance and chrominance blocks may be filtered, only luminance blocks may be filtered, outer boundary pixels may be filtered, and only block border pixels may be filtered.

\section{RESULTS}

In [2] the performance of the adaptive filter, only de-blocking and de-ringing part, was evaluated against using no post filtering and filtering as recommended in H.263 App. III [5]. It was shown that the adaptive filter improve visual quality by combat both de-blocking and de-ringing artifacts and also that the peak signal-to-noise ratio (PSNR) was comparable with the results from using the H.263 
App. III filter. Here the adaptive filter is evaluated by examining the PSNR value and the perceptual quality both against; No filtering, only de-blocking and de-ringing. The algorithms are processed on decoded H.263 profile 0 bit streams for two different sequences each presented at four different bit rates at 15 frames per second (fps) and of size $176 \times 144(\mathrm{QCIF})$. The size, bit-rates and frame rate are chosen to correspond to the use in todays $2 \mathrm{G}$ and $3 \mathrm{G}$ networks. The PSNR is calculated for the post processed images and an average for the complete sequence. The PSNR of an $M \times N$ image is given by

$$
P S N R=10 \log \frac{M N \times 255^{2}}{\sum_{m, n}\left\|f(m, n)-f_{\text {org }(m, n)}\right\|^{2}}
$$

The sequence used is "Foreman" and the results are shown in Table 1. In the table it is shown that the adaptive filter

\begin{tabular}{|c|c|c|}
\hline \multicolumn{3}{|c|}{ Foreman } \\
\hline $\begin{array}{l}\text { Bitrate } \\
{[\mathrm{kbit} / \mathrm{s}]}\end{array}$ & Filter & $\begin{array}{l}\text { Average } \\
\text { PSNR [dB] } \\
\text { for YUV }\end{array}$ \\
\hline 48 & No Post Filter & 33.078 \\
\hline 48 & Adaptive Post Filter [2] & 33.129 \\
\hline 48 & Proposed Adaptive Filter & 33.077 \\
\hline 64 & No Post Filter & 33.481 \\
\hline 64 & Adaptive Post Filter [2] & 33.548 \\
\hline 64 & Proposed Adaptive Filter & 33.478 \\
\hline 128 & No Post Filter & 35.795 \\
\hline 128 & Adaptive Post Filter [2] & 35.875 \\
\hline 128 & Proposed Adaptive Filter & 35.138 \\
\hline 196 & No Post Filter & 37.508 \\
\hline 196 & Adaptive Post Filter [2] & 37.562 \\
\hline 196 & Proposed Adaptive Filter & 36.442 \\
\hline
\end{tabular}

Table 1. Results from de-blocking and de-ringing on Foreman. All sequences have a QCIF resolution and $15 \mathrm{fps}$.

always keeps the PSNR for low bit-rates compared to the original decoded sequences and that the PSNR slightly decreases when the amount the sharpening increases which is notified for the higher bit-rates. This decrease is expected since the PSNR is a measure of similarity to the original image, and the sharpening is not always restoring the original values even when the perceptual quality is increased, which is visualized in Fig. 5-6. In Fig. 5 there is very little sharpening performed and therefore almost no visible effects can be seen. In Fig. 6 the sharpening effects is more obvious and there is an increase of perceptual quality even though the decrease of PSNR.

\section{CONCLUSION}

This paper has described an adaptive filter that can remove de-blocking and de-ringing artifacts and also be used to enhance the sharpness of decoded video, which may be caused by zeroing high-frequency DCT coefficients. The adaptive filters described here uses the reference filter output to control the filter function, which gives a low computational power and memory consumption.

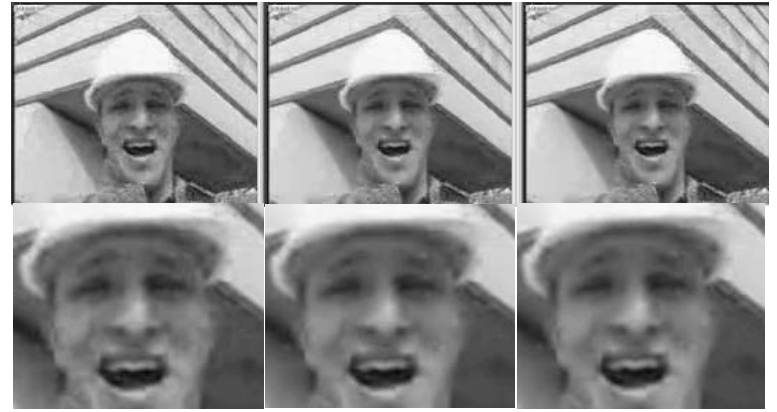

Fig. 5. Luminance output from Foreman in QCIF format, coded at 64 kbps and 15 fps. From left, No Post Filter, Adaptive Post Filter [2], and Proposed Adaptive Filter.

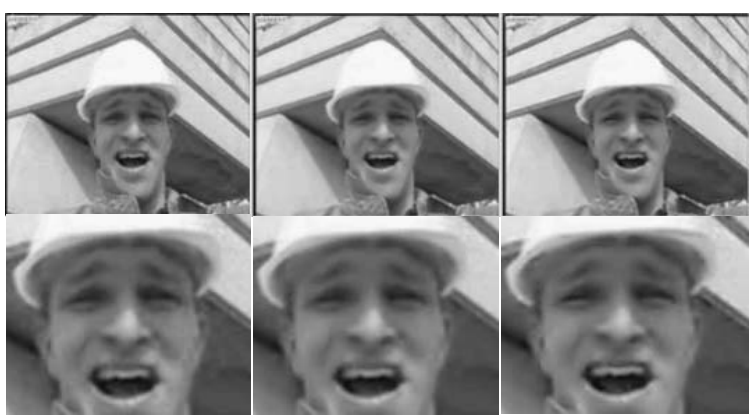

Fig. 6. Luminance output from Foreman in QCIF format, coded at 196 kbps and 15 fps. From left, No Post Filter, Adaptive Post Filter [2], and Proposed Adaptive Filter

\section{REFERENCES}

[1] A. Rossholm and K. Andersson, "Adaptive Deblocking De-ringing Filter," IEEE International Conference on Image Processing 2005, pp. 1042-5., Genoa, Italy, 2005.

[2] M. Yuen, H. R. Wu, "A survey of hybrid MC/DPCM/DCT video coding distortions," Signal Processing, vol. 70, pp. 247-278, July 1998.

[3] H. C. Reeve III, Jae S. Lim, "Reduction of Blocking Effect in Image Coding," Proc. ICASSP, pp. 12121215, Boston, Mass. 1983.

[4] US patent No. 5,488,420 to G. Bjontegaard for "Cosmetic filter for smoothing regenereted pictures, , e.g. after Signal Compression for Transmission in a Narrowband Network".

[5] ITU-T Recommendation H.263 Appendix III: "Examples for H.263 Encoder/Decoder Implementations," June 2000.

[6] G. Scognamiglioa, G Ramponia, A. Rizzi, "Enhancement of coded video sequences via and adaptive nonlinear post- processing," Image Communications, vol. 18, pp. 127-139, 2003 